\title{
Does Pol I talk to Pol II? Coordination of RNA polymerases in ribosome biogenesis
}

\author{
Annemieke A. Michels and Nouria Hernandez ${ }^{1}$ \\ Center for Integrative Genomics, University of Lausanne, CH-1015 Lausanne, Switzerland
}

To grow and to divide, cells are dependent on protein synthesis, and protein synthesis depends on ribosomes. Ribosomes are complex molecular machines that contain four different RNA molecules and 79 different proteins, produced by three different RNA polymerases. RNA polymerase I (Pol I) synthesizes the large ribosomal RNA (rRNA) precursor (35S RNA in yeast), which is then processed into mature 18S, 28S, and 5.8S RNAs; RNA polymerase II (Pol II) synthesizes the mRNAs encoding the ribosomal proteins; and RNA polymerase III (Pol III) synthesizes the small 5S RNA molecule. The biosynthesis of ribosomes by the three RNA polymerases uses an enormous amount of the cell resources. In a yeast cell, rRNA transcription represents $\sim 60 \%$ of total transcription, and transcription of the ribosomal protein mRNAs represents $\sim 50 \%$ of all Pol II transcription initiation events (Warner 1999; Rudra and Warner 2004). Accordingly, ribosome biosynthesis is tightly regulated with cell growth and proliferation. Thus, when the demand for protein synthesis is reduced, as occurs with cells in stationary phase after nutrient deprivation, the production of new ribosomes is greatly reduced.

Ribosome assembly requires the coordinated production of ribosome components. On the one hand, equimolar amounts of each ribosome protein must be produced. In yeast, the coregulation of ribosomal protein synthesis is exerted mainly at the level of ribosomal protein gene transcription. On the other hand, sufficient amounts of each ribosomal protein must be produced to allow correct assembly and processing of rRNAs. This suggests that the ribosomal protein and rRNA synthesis machineries are somehow coregulated. Indeed, an essential signal transduction pathway for the modulation of rRNA, ribosomal proteins, and 5S rRNA in response to nutrient availability is the TOR (Target of Rapamycin) kinase pathway. This pathway is activated in the presence of nutrients, and inactivation of TOR-for example, by the antibiotic rapamycin-mimics the effects of starvation (for review, see Crespo and Hall 2002). Rapamycin inhibition of the TOR pathway is known to repress rRNA transcription by Pol I, Pol III transcription, and Pol II transcription of ribosomal protein genes (Zaragoza et

${ }^{1}$ Corresponding author.

E-MAIL Nouria.Hernandez@unil.ch; FAX 41-21-692-39-25.

Article is online at http://www.genesdev.org/cgi/doi/10.1101/gad.1460706. al. 1998; Powers and Walter 1999). However, we still know little about how TOR controls ribosome component synthesis, in particular, whether each of the three RNA polymerase machineries is controlled separately, whether one of them is the primary target that then controls the others, or whether several mechanisms of crosstalk ensure coordinated regulation.

In this issue of Genes \& Development, Laferté et al. (2006) present data suggesting that in yeast, accumulation of large ribosomal RNAs as a result of deregulated Pol I transcription leads to a corresponding accumulation of ribosomal proteins, 5S rRNA, and fully assembled ribosomes. Thus, increased Pol I transcription activity can orchestrate the coordinated increased accumulation of all ribosomal components, suggesting a central role for RNA polymerase I activity in the coordination of ribosomal component synthesis.

Transcription by Pol I in yeast is known to depend on four factors: Three of them-TBP (TATA-box binding protein) and the multisubunit complexes UAF (upstream activating factor) and CF (core factor)-bind together to rDNA promoters; the fourth, Rrn3, associates reversibly with Pol I and renders it competent for transcription initiation, at least in part by bridging Pol I and the promoter-bound factors (for review, see Moss and Stefanovsky 2002; Grummt 2003; Russell and Zomerdijk 2006). The association of Rrn3 with Pol I controls the activity of the enzyme, and the percentage of active Pol I in the cell appears in turn to play a determinant role in the control of rRNA transcription. The investigators created a yeast strain, which they named CARA (for $\underline{\text { Constitutive Asso- }}$ ciation of $\underline{\operatorname{R}} r \mathrm{3}$ and $\underline{\mathrm{A}} 43$ ), in which the endogenous genes coding for Rrn3 and A43, the Pol I subunit with which Rrn3 is known to associate (Peyroche et al. 2000), were deleted. Rrn3 and A43 activities were supplied as a fusion protein expressed from a $2-\mu$ plasmid. The fusion protein assembled properly with the other Pol I subunits to form a constitutively active enzyme. Remarkably, under normal growth conditions, the CARA strain behaves like the wild type: It has the same doubling time and the same amount of ribosomal particles, and it has a similar mRNA expression pattern. Thus, the CARA strain has a normal physiology.

Modulation of Pol I transcription through TOR targets Rrn3. Upon rapamycin treatment, Rrn3 dissociates from the Pol I complex, resulting in an arrest of transcription 
(Milkereit and Tschochner 1998; Miller et al. 2001; Grummt 2003). In the CARA strain, however, the Rrn3A43 fusion protein is not dissociable, and Pol I transcription was much less affected. The decrease of $35 \mathrm{~S}$ transcription after rapamycin treatment was greatly delayed, and chromatin immunoprecipitation (ChIP) analysis revealed an attenuation of the decrease of Pol I molecules on 35 S rDNA. Actin transcription was unaffected, showing the specificity of the system. The CARA strain offered, then, an excellent opportunity to investigate the consequences of deregulated Pol I transcription on synthesis of the other ribosomal components.

The effects on $5 \mathrm{~S}$ rRNA were monitored by a relatively long pulse labeling with $\left[{ }^{3} \mathrm{H}\right]$ uracil, which indicated that after rapamycin treatment, 5S accumulation was much less reduced in CARA cells as compared to normal cells. This was consistent with an effect on Pol III transcription; however, a ChIP analysis failed to show an increased Pol III occupancy of 5S rDNA genes in CARA cells relative to wild-type cells, suggesting that the increased 5S rRNA levels result from increased stabilization of the $5 \mathrm{~S}$ transcript.

What about the other components of ribosomes, the ribosomal proteins? A microarray analysis revealed that CARA cells have a similar mRNA expression pattern as wild-type cells in the absence of rapamycin. Strikingly, after rapamycin treatment, all mRNAs were similarly down-regulated in wild-type and CARA cells, except for the mRNAs encoding ribosomal proteins. Thus, 147 mRNAs, accounting for $2.5 \%$ of the mRNAs analyzed, were over-represented in the CARA strain after rapamycin treatment, 128 of which encode ribosomal proteins! The results were confirmed for four ribosomal protein mRNAs by Northern blots and primer extensions. Moreover, ChIP analysis revealed an increased Pol II occupancy on the two ribosomal protein genes tested, whereas the Pol II genes ACT1 and ADH1 were unaffected. Deregulation of Pol I, then, specifically affects transcription of a subset of Pol II genes, those encoding ribosomal proteins.

Since after rapamycin treatment CARA cells contain higher levels of all ribosome components as compared to wild-type cells, they might also contain more ribosomes. Indeed, whereas under normal growth conditions both strains contained similar amounts of 405 and 605 ribosomal particles, upon rapamycin treatment 40S and 60S particle levels decreased much more slowly in CARA cells, such that after $180 \mathrm{~min}$ of treatment these cells contained twice as many $40 \mathrm{~S}$ and 60S particles as wildtype cells. Thus, the diminished repression of Pol I transcription after rapamycin treatment in CARA cells leads to a smaller decrease in ribosomes.

Are all these effects restricted to cells treated with rapamycin? Because in stationary cells Rrn3 is largely dissociated from Pol I and Pol I transcription is repressed (Milkereit and Tschochner 1998), the investigators chose to address this question by examining CARA cells having undergone the diauxic shift. As after rapamycin treatment, the decrease in Pol I transcription and in levels of $5 \mathrm{~S}$ rRNA was attenuated relative to wild-type cells. Moreover, whereas CARA and wild-type cells grown to mid-log phase had a similar expression of Pol II mRNAs as evaluated by microarray analysis, ribosomal protein mRNAs were overrepresented in the CARA strain after the diauxic shift. Thus, CARA cells behave similarly after rapamycin treatment and under physiological conditions normally leading to Pol I transcription repression.

Together, the results indicate that deregulation of Pol I transcription, specifically an attenuation of Pol I transcription repression, leads to a concomitant attenuation of 5S rRNA and ribosomal protein mRNA decrease. The relatively higher level of $5 \mathrm{~S}$ rRNA after rapamycin treatment might result from increased stabilization of $5 \mathrm{~S}$ rRNA as a result of incorporation into increased numbers of ribosomes. This implies that $5 \mathrm{~S}$ rRNA is produced in excess relative to other ribosomal components, at least in CARA cells after rapamycin treatment. On the other hand, the relative higher levels of ribosomal protein mRNAs apparently result from higher Pol II transcription of ribosomal protein genes, as evidenced by Pol II occupancy of these genes. This raises the question of how activated Pol I transcription signals specifically to ribosomal protein gene Pol II transcription. Laferté et al. (2006) mention several possibilities: a sensing of the Pol I transcription product, the $35 \mathrm{~S}$ transcripts; a sensing of the Pol I transcription process itself, through the sensing of, for example, activated Pol I promoters or the titration of molecules involved in transcription; and the possibility that activation of Pol II ribosomal genes might be linked to the integrity of the nucleolus, which itself depends on active Pol I transcription (for review, see Mayer and Grummt 2005). This last hypothesis would be more plausible if active Pol II ribosomal protein genes were located close to nucleoli, an interesting possibility given the accumulating evidence that transcription of class II genes depends on their nuclear localization (Dernburg et al. 1996; Gerasimova et al. 2000; Feuerbach et al. 2002; Cabal et al. 2006). It is also conceivable that noncoding RNA derived from the intergenic spacers of rRNA repeats, which have recently been shown to play a role in recruiting NoRC, a chromatin-remodeling machine involved in rRNA repression (Mayer et al. 2006), somehow mediate the Pol I-Pol II ribosomal protein gene transcription cross-talk.

Figure 1 summarizes some aspects of ribosomal component regulation by rapamycin. For Pol I transcription, TOR is known to activate Rrn3 and to allow its association with Pol I, and rapamycin inhibition of TOR results in dissociation of Rrn3 from Pol I (Claypool et al. 2004). The mechanistic details of this effect are not known in yeast, but in mouse cells, the rapamycin effects on TIF1A-the mouse equivalent of Rrn3-have been studied in some detail. In such cells, inhibition of TOR, itself an inhibitor of PP2A, results in dephosphorylation of TIF$1 \mathrm{~A}$ serine 44 and inactivation of the factor. Moreover, rapamycin treatment results in phosphorylation of TIF1A serine 199, which results in dissociation of TIF-1A from Pol I. TIF-1A then becomes mainly cytoplasmic and Pol I transcription is repressed (Mayer et al. 2004). 


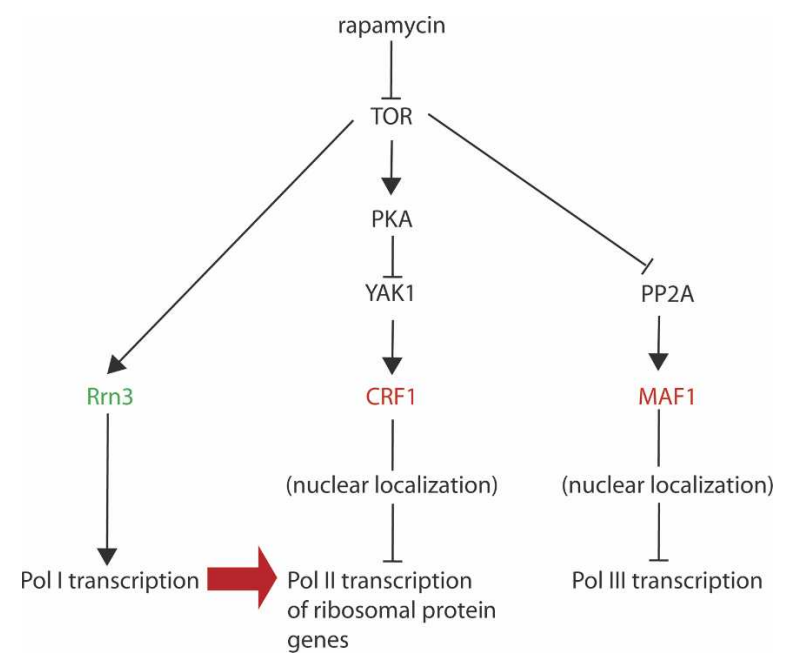

Figure 1. Three examples of pathways by which rapamycin down-regulates synthesis of ribosomal components. Many others exist that are not depicted here. The arrows do not necessarily indicate a direct effect. Rapamycin represses TOR. For Pol I transcription, this prevents TOR activation of Rrn3, which binds to Pol I and renders it competent for transcription initiation. In mouse cells, rapamycin treatment prevents TIF-1A association with Pol I and causes it to become largely cytoplasmic. For Pol II transcription, inhibition of TOR prevents activation of PKA, itself a repressor of YAK1. When derepressed, YAK1 phosphorylates CRF1, which translocates into the nucleus and inhibits Pol II transcription of ribosomal protein genes. For Pol III transcription, inhibition of TOR prevents its repressive effect on PP2A. When derepressed, PP2A phosphorylates Mafl, which translocates into the nucleus and represses Pol III transcription, not only of 5S rRNA but also of all other Pol III genes.

Thus, in the case of Pol I, rapamycin treatment inactivates a positive Pol I transcription factor.

Pol II transcription of ribosomal RNA genes is also controlled by TOR. In yeast, the proteins Fhl1, Ifh1, and Crf1 are key regulators of ribosomal protein mRNA synthesis. Fhll associates on ribosomal protein gene promoters with either Ifh 1 to activate ribosomal protein gene transcription or Crf1 to repress transcription (Martin et al. 2004). TOR, via PKA, negatively regulates the kinase YAK1 and thus maintains Crf1 in the cytoplasm. Upon rapamycin-mediated inactivation of TOR, YAK1 becomes activated and phosphorylates Crf1, which accumulates in the nucleus and represses transcription of ribosomal protein genes by competing with Ifh 1 for binding to Fhll at ribosomal protein gene promoters (Martin et al. 2004).

In the case of Pol III transcription, rapamycin inhibition occurs through Maf1. The MAF1 gene was originally identified in Saccharomyces cerevisiae by the isolation of a temperature-sensitive mutation, maf1-1, which affected tRNA suppressor efficiency and interacted genetically with Pol III (Murawski et al. 1994). It was then shown that in maf1-1 cells, Pol III transcription was elevated (Pluta et al. 2001), consistent with Maf1 being a repressor of Pol III transcription. Indeed, further work characterized Maf1 as a common component of multiple signaling pathways that repress Pol III transcription; in particular, rapamycin-induced repression of Pol III transcription was Maf1-dependent (Upadhya et al. 2002). Very recent results have shown that Maf1 is a repressor of all Pol III transcription. Upon rapamycin treatment, Maf1 is dephosphorylated by PP2A, translocates into the nucleus, and represses Pol III transcription (OficjalskaPham et al. 2006; Roberts et al. 2006).

The Laferté et al. work presented in this issue of Genes \& Development allows us to add an arrow to this model, indicating that changes in Pol I transcription can dictate changes in Pol II transcription of the ribosomal protein genes. Is Pol II transcription activity on ribosomal protein genes, then, subordinated to Pol I activity, or can Pol II activity on ribosomal protein genes also influence Pol I transcription activity? Some evidence in support of the latter comes from experiments involving Fhl1 or Ifh1 (Rudra et al. 2005). As mentioned above, Fhll and Ifh1 proteins are key regulators of ribosomal protein mRNA synthesis by Pol II in yeast (Martin et al. 2004). Cells lacking Fhl1 or both Fhl1 and Ifh1 proteins contain less than one-quarter of the normal amount of ribosomes and are deficient not only for transcription of ribosomal protein genes but also for general Pol II transcription. Moreover, production of rRNA by Pol I is reduced. However, since in these cells all Pol II transcription is reduced, it is difficult to conclude that this effect results from specific cross-talk between Pol II ribosomal protein gene transcription and Pol I transcription. It is not clear, then, that Pol II activity on ribosomal protein genes can dictate changes in Pol I activity. Nevertheless, it is possible that the direction of the cross-talk between Pol I and Pol II changes depending on which one of the activities is upor down-regulated. Thus, when Pol I is up-regulated (or less repressed), it may be crucial to ensure that a matching set of ribosomal proteins be synthesized, so as to ensure proper large rRNA processing and assembly. For the same reasons, one might imagine that up-regulation of the Pol II ribosomal protein genes by a method such as, perhaps, fusing Fhl1 to the activator Ifh1, might in turn demand increased Pol I transcription.

An important question raised by this work is whether a similar cross-talk between Pol I and Pol II transcription of ribosomal protein genes can be evidenced in mammalian cells. In such cells, the main mechanism by which TOR regulates the production of ribosomal proteins is at the level of translation rather than transcription, through the S6 kinase (Thomas and Hall 1997; Ruggero and Pandolfi 2003). Nevertheless, it is highly possible that the mechanism linking Pol I transcription to Pol II transcription activity at ribosomal protein genes has been conserved. Given the consistent overexpression of ribosomal components in cancer cells, this is a hypothesis worth testing.

\section{References}

Cabal, G.G., Genovesio, A., Rodriguez-Navarro, S., Zimmer, C., Gadal, O., Lesne, A., Buc, H., Feuerbach-Fournier, F., Olivo- 
Marin, J.C., Hurt, E.C., et al. 2006. SAGA interacting factors confine sub-diffusion of transcribed genes to the nuclear envelope. Nature 441: 770-773.

Claypool, J.A., French, S.L., Johzuka, K., Eliason, K., Vu, L., Dodd, J.A., Beyer, A.L., and Nomura, M. 2004. Tor pathway regulates Rrn3p-dependent recruitment of yeast RNA polymerase I to the promoter but does not participate in alteration of the number of active genes. Mol. Biol. Cell 15: 946956.

Crespo, J.L. and Hall, M.N. 2002. Elucidating TOR signaling and rapamycin action: Lessons from Saccharomyces cerevisiae. Microbiol. Mol. Biol. Rev. 66: 579-591.

Dernburg, A.F., Broman, K.W., Fung, J.C., Marshall, W.F., Philips, J., Agard, D.A., and Sedat, J.W. 1996. Perturbation of nuclear architecture by long-distance chromosome interactions. Cell 85: 745-759.

Feuerbach, F., Galy, V., Trelles-Sticken, E., Fromont-Racine, M., Jacquier, A., Gilson, E., Olivo-Marin, J.C., Scherthan, H., and Nehrbass, U. 2002. Nuclear architecture and spatial positioning help establish transcriptional states of telomeres in yeast. Nat. Cell Biol. 4: 214-221.

Gerasimova, T.I., Byrd, K., and Corces, V.G. 2000. A chromatin insulator determines the nuclear localization of DNA. Mol. Cell 6: 1025-1035.

Grummt, I. 2003. Life on a planet of its own: Regulation of RNA polymerase I transcription in the nucleolus. Genes \& Dev. 17: 1691-1702.

Laferté, A., Favry, E., Sentenac, A., Riva, M., Carles, C., and Chédin, S. 2006. The transcriptional activity of RNA polymerase I is a key determinant for the level of all ribosome components. Genes \& Dev. (this issue).

Martin, D.E., Soulard, A., and Hall, M.N. 2004. TOR regulates ribosomal protein gene expression via PKA and the Forkhead transcription factor FHL1. Cell 119: 969-979.

Mayer, C. and Grummt, I. 2005. Cellular stress and nucleolar function. Cell Cycle 4: 1036-1038.

Mayer, C., Zhao, J., Yuan, X., and Grummt, I. 2004. mTORdependent activation of the transcription factor TIF-IA links rRNA synthesis to nutrient availability. Genes \& Dev. 18: 423-434.

Mayer, C., Schmitz, K.M., Li, J., Grummt, I., and Santoro, R. 2006. Intergenic transcripts regulate the epigenetic state of rRNA genes. Mol. Cell 22: 351-361.

Milkereit, P. and Tschochner, H. 1998. A specialized form of RNA polymerase I, essential for initiation and growth-dependent regulation of rRNA synthesis, is disrupted during transcription. EMBO I. 17: 3692-3703.

Miller, G., Panov, K.I., Friedrich, J.K., Trinkle-Mulcahy, L., Lamond, A.I., and Zomerdijk, J.C. 2001. hRRN3 is essential in the SL1-mediated recruitment of RNA Polymerase I to rRNA gene promoters. EMBO I. 20: 1373-1382.

Moss, T. and Stefanovsky, V.Y. 2002. At the center of eukaryotic life. Cell 109: 545-548.

Murawski, M., Szczesniak, B., Zoladek, T., Hopper, A.K., Martin, N.C., and Boguta, M. 1994. mafl mutation alters the subcellular localization of the Mod5 protein in yeast. Acta Biochim. Pol. 41: 441-448.

Oficjalska-Pham, D., Harismendy, O., Smagowicz, W.J., Gonzalez de Peredo, A., Boguta, M., Sentenac, A., and Lefebvre, O. 2006. General repression of RNA polymerase III transcription is triggered by protein phosphatase type 2A-mediated dephosphorylation of Maf1. Mol. Cell 22: 623-632.

Peyroche, G., Milkereit, P., Bischler, N., Tschochner, H., Schultz, P., Sentenac, A., Carles, C., and Riva, M. 2000. The recruitment of RNA polymerase I on rDNA is mediated by the interaction of the A43 subunit with Rrn3. EMBO J. 19:
5473-5482.

Pluta, K., Lefebvre, O., Martin, N.C., Smagowicz, W.J., Stanford, D.R., Ellis, S.R., Hopper, A.K., Sentenac, A., and Boguta, M. 2001. Maf1p, a negative effector of RNA polymerase III in Saccharomyces cerevisiae. Mol. Cell. Biol. 21: 5031-5040.

Powers, T. and Walter, P. 1999. Regulation of ribosome biogenesis by the rapamycin-sensitive TOR-signaling pathway in Saccharomyces cerevisiae. Mol. Biol. Cell 10: 987-1000.

Roberts, D.N., Wilson, B., Huff, J.T., Stewart, A.J., and Cairns, B.R. 2006. Dephosphorylation and genome-wide association of Maf1 with Pol III-transcribed genes during repression. Mol. Cell 22: 633-644.

Rudra, D. and Warner, J.R. 2004. What better measure than ribosome synthesis? Genes \& Dev. 18: 2431-2436.

Rudra, D., Zhao, Y., and Warner, J.R. 2005. Central role of Ifh $1 \mathrm{p}-\mathrm{Fhl1p}$ interaction in the synthesis of yeast ribosomal proteins. $E M B O$ J. 24: 533-542.

Ruggero, D. and Pandolfi, P.P. 2003. Does the ribosome translate cancer? Nat. Rev. Cancer 3: 179-192.

Russell, J. and Zomerdijk, J.C. 2006. The RNA polymerase I transcription machinery. Biochem. Soc. Symp. (73): 203 216.

Thomas, G. and Hall, M.N. 1997. TOR signalling and control of cell growth. Curr. Opin. Cell Biol. 9: 782-787.

Upadhya, R., Lee, J., and Willis, I.M. 2002. Maf1 is an essential mediator of diverse signals that repress RNA polymerase III transcription. Mol. Cell 10: 1489-1494.

Warner, J.R. 1999. The economics of ribosome biosynthesis in yeast. Trends Biochem. Sci. 24: 437-440.

Zaragoza, D., Ghavidel, A., Heitman, J., and Schultz, M.C. 1998. Rapamycin induces the $G_{0}$ program of transcriptional repression in yeast by interfering with the TOR signaling pathway. Mol. Cell. Biol. 18: 4463-4470. 


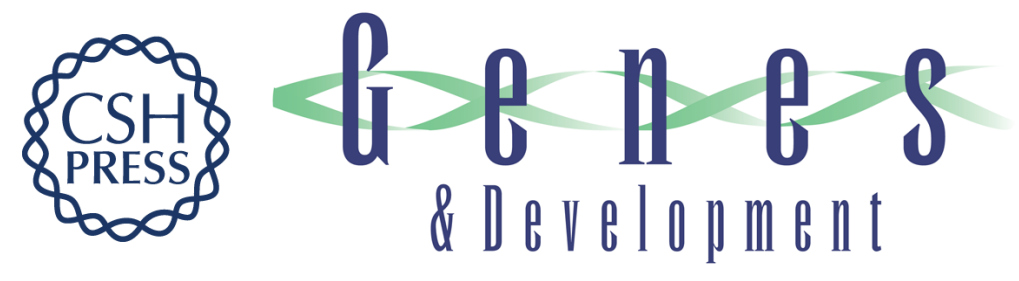

\section{Does Pol I talk to Pol II? Coordination of RNA polymerases in ribosome biogenesis}

Annemieke A. Michels and Nouria Hernandez

Genes Dev. 2006, 20:

Access the most recent version at doi:10.1101/gad.1460706 $\begin{array}{cl}\text { Related Content } & \begin{array}{l}\text { The transcriptional activity of RNA polymerase I is a key determinant for the level of } \\ \text { all ribosome components } \\ \text { Arnaud Laferté, Emmanuel Favry, André Sentenac, et al. } \\ \text { Genes Dev. UNKNOWN, 2006 20:2030-2040 }\end{array} \\ \text { References } & \begin{array}{l}\text { This article cites } 28 \text { articles, } 13 \text { of which can be accessed free at: } \\ \text { http://genesdev.cshlp.org/content/20/15/1982.full.html\#ref-list-1 }\end{array} \\ & \begin{array}{l}\text { Articles cited in: } \\ \text { http://genesdev.cshlp.org/content/20/15/1982.full.html\#related-urls }\end{array} \\ \text { License } & \begin{array}{l}\text { Receive free email alerts when new articles cite this article - sign up in the box at the top } \\ \text { right corner of the article or click here. }\end{array}\end{array}$

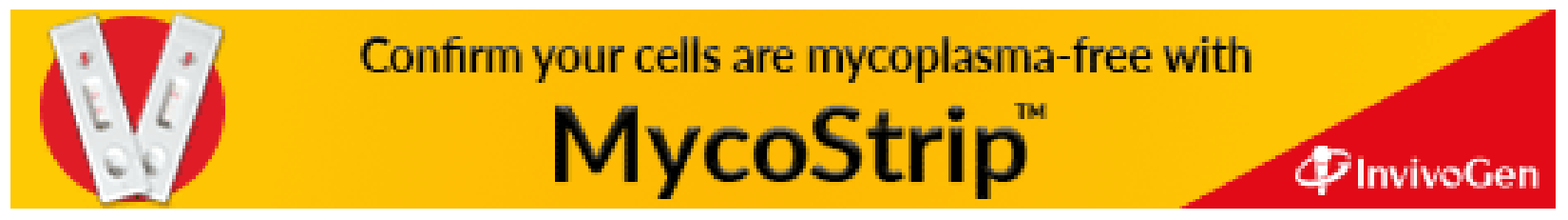

